\title{
Latest developments in innovative manufacturing to combine nanotechnology with healthcare
}

\author{
"To fully harness the many benefits of nanotechnology in \\ healthcare, innovative manufacturing is necessary to mass \\ produce nanoparticles and nanofibers, the two major types \\ of nanofeatures currently sought after and of immense \\ utilitarian value in healthcare."
}

\author{
Maryam Parhizkar ${ }^{1}$, Suntharavathanan Mahalingam', Shervanthi \\ Homer-Vanniasinkam ${ }^{1,2,3}$ \& Mohan Edirisinghe ${ }^{*, 1}$ \\ 'Department of Mechanical Engineering, University College London, Torrington Place, London WC1E 7JE, UK \\ 'Leeds Vascular Institute Leeds General Infirmary, Great George Street, Leeds LS1 3EX, UK \\ ${ }^{3}$ Division of Surgery, University of Warwick \& University Hospitals Coventry \& Warwickshire NHS Trust, Clifford Bridge \\ Rd, Coventry CV2 2DX, UK \\ *Author for correspondence: m.edirisinghe@ucl.ac.uk
}

Keywords: healthcare $\bullet$ manufacturing $\bullet$ nanofibers $\bullet$ nanoparticles $\bullet$ nanotechnology

First draft submitted: 12 September 2017; Accepted for publication: 27 September 2017; Published online: 29 November 2017

Nanotechnology has become increasingly important in advancing the frontiers of many key areas of healthcare, for example, drug delivery and tissue engineering. To fully harness the many benefits of nanotechnology in healthcare, innovative manufacturing is necessary to mass produce nanoparticles and nanofibers, the two major types of nanofeatures currently sought after and of immense utilitarian value in healthcare. For example, nanoparticles are a key drug delivery enabler, the structural and mechanical mimicry are important attributes of nanofiber which are increasingly used as biomimetic agents.

\section{Nanoparticles \& innovative manufacturing}

It is essential to understand the process of nanoparticle generation in order to achieve the desired particles. Polymeric nanoparticles have gained immense interest as therapeutic carriers capable of loading, protecting and targeting drugs/genes to desired sites. Several techniques have been developed to generate nanoparticles. Particle characteristics such as size, structure and morphology differ depending on the method of preparation. Different methods also enable the modification of drug loading, drug encapsulation efficiency and release kinetics. At the same time, production methods that are cost effective and reproducible on an industrial scale are preferred in the selection criteria and in this context electrospraying is evolving as the formidable scalable process.

Also referred to as electrohyrodynamic atomization (EHDA), electrospraying is a method of fabrication of micro- and nano-sized particles by the application of external electric force [1] This is a key method which shows real manufacturing promise on a large scale as it can be multiplexed. Basically, the solution containing the drug and polymer is infused through a nozzle and a high voltage ( $\mathrm{kV}$ range) is applied; the solution is then electrically charged and a jet is formed that breaks into smaller droplets. The droplets undergo further reduction in

Future $\because$ Medicine part of 
size with evaporation of the solvent and ultimately particles with defined size and shape are produced. Particle size and morphology can be controlled by adjusting the solution properties (i.e., concentration, viscosity, surface tension, electrical conductivity and evaporation rate of liquid) and processing parameters (flow rate and applied voltage and distance at which particles are collected). Electrospray production of particles has numerous advantages, from high drug loading efficiency to narrow particle size distribution. The generation of particles via electrospraying is a single-step process that does not require further particle drying processes (such as freeze drying), which could damage the particle morphology as well as the encapsulated drug.

EHDA facilitates encapsulation of both hydrophobic and hydrophilic drugs with high encapsulation efficiency, also offering exceptional control over particle size and size distribution in both the nano and micro length scales. One of the key areas that attracted electrohydrodynamic encapsulation of drugs is in cancer treatment. Targeted delivery of potent, toxic chemotherapy drugs requires precise control over encapsulating them in polymeric nano carriers. Although other conventional processing methods were previously used to prepare the drug cisplatin loaded carriers [2], the EHDA process was first used systematically by Parhizkar et al. [3] to fabricate cisplatin-loaded particles with high drug encapsulation efficiencies. In the study, cisplatin was successfully encapsulated with high efficiency $(>70 \%)$ in poly (lactic-co-glycolic acid) polymeric nanoparticles. Three different drug:polymer ratios (2.5, 5 and $10 \mathrm{wt} \%$ cisplatin) were electrosprayed and particles with mean diameter of $550 \pm 80 \mathrm{~nm}$ were produced with the highest drug:polymer ratio possessing the best sustained release (smallest burst release). The control over drug dosage and period of release afforded by cisplatin encapsulation that was facilitated by the EHDA technique is essential in delivery of cytotoxic chemotherapy agents.

More complex configurations of EHDA have been proposed as proof of versatility of this technique. Coaxial EHDA, comprising two concentric capillaries, is a good example. Reardon et al. [4] produced core-shell structures facilitated by coaxial EHDA and a comparison study on the role of particle architecture on the rate of cisplatin release was successfully conducted. Cellular uptake and cytotoxicity efficacy of the drug-loaded particles were examined on a human head and neck squamous carcinoma cell line. It was demonstrated that nanoparticles were effectively internalized into the endolysosomal compartments of cancer cells and an increased cytotoxic efficacy was observed compared with free drug in vitro.

Coaxial electrohydrodynamic atomization as well as multiple capillary systems (i.e., 3-needle or 4-needle EHDA) enables the simultaneous use of two or more immiscible fluids and as a result encapsulation of multidrug and polymers with different hydrophobic/hydrophilic characteristics [5]. Multidrug systems can immensely benefit from this technique that can provide control of the individual drug release profile by designing the surface properties of particles without impairing the drug within the core. Multilayer particles produced by this technique can preserve the bioactivity of unstable biomolecules. Although the production rate of particles with EHDA technique is low, multiplex electrospray setups can offer a new route to industrial scale production of particles [6].

\section{Nanofibers \& innovative manufacturing}

Nanofibers are a versatile class of nanomaterials that has attracted increasing attention in healthcare applications in recent years. Important biomedical applications of nanofibers include filtration, medical textiles, wound healing patches, tissue engineering/regeneration scaffolds, biosensors and drug delivery tampons. Nanofibers made of biocompatible or biodegradable materials show tremendous potential in the healthcare sector due to their unique combination of intrinsic functionalities and properties. For example, high surface area and high surface and interior porosity associated with nanofibers that enhance adhesion of cells as well as different proteins and drug molecules are key attributes, which make them superior to their micro/macro counterparts. Major classes of nanofibers used in healthcare applications are prepared from various natural and synthetic polymers and their composites. Ceramic/inorganic nanofibers including carbon-based fibers are also increasingly being used. The largest market share of nanofibers for biomedical applications is accounted for by filtration followed by medical textiles, wound healing, tissue engineering. In the next few years, nanofiber consumption is expected to show considerable growth. But the crucial challenge is the availability of robust and homogeneous nanofibers in sufficient quantities. Industrial developments in manufacturing technology adopting various proprietary methods based on electrospinning, traditional centrifugal spinning, solution and melt spinning and melt-blowing methods have provided impetus for realizing the commercial potential of nanofibers but cannot mass produce sufficient quantities and have essentially become batch processes [7].

Pressurized gyration [8] and its sister-manufacturing routes invented since 2013 offer convenient and versatile techniques to prepare nanofibers and nanofibrous structures in a single step on a large scale. Basically, it utilizes 
the simultaneous application of high rotating speed and working pressure to spin nanofibers and nanofibrous structures essentially from a perforated aluminum pot containing a polymer solution/suspension. In a typical pressurized gyration process the combination of centrifugal and dynamic fluid flow forces overcomes the surface tension force to generate fibers. The liquid jet ejected from the holes of the gyration vessel undergoes an instability at the liquid-air interface to generate nanofibers. At the final stage of the process, the generated liquid jet solidifies to form the nanofibers at the collector. This solidification depends on the solvent properties such as evaporation rate and the collector-gyration vessel distance.

In the last few years pressurized gyration variants have become promising alternative methods of fiber spinning, overcoming the drawbacks of other spinning methods and have attained wide popularity in the manufacturing and healthcare communities. Several useful properties could be achieved with these nanofibers such as a high surface area to volume ratio, flexibility and surface functional properties. It makes use of centrifugal spinning and solution blowing concomitantly to generate both simple/complex architectures such as homogenous nanofibers, blended nanofibers, nanoparticle-loaded nanofibers, bubbles, capsules and biomolecules-containing nanofibers in large quantities. The size, size distribution and morphology of these architectures could be varied by simple adjustment of concentration of the solution/suspension, rotating speed and the working pressure. It has been possible to extend pressurized gyration into different forms, each is unique and very recent inventions are pressure-coupled infusion gyration, pressurized melt gyration and infusion gyration where the flow of the polymer solution instead of applied pressure is continuous and controlled. Thus, in addition to rotating speed, the flow rate of the feedstock becomes a key process control parameter. On the other hand, in pressurized melt gyration, a polymer melt is used instead of liquid to generate products. Here polymer pellets in the gyration vessel are melted using input heat. The most recent development involves pressure-coupled infusion gyration, where working pressure has been applied to a gyrating vessel in addition to controlling the flow of polymer solution. This simple and efficient process allows parallel formation of a multitude of different fibrous architectures with regular morphology, even using polymer concentrations that are normally difficult to spin.

Other healthcare-significant products containing nanostructures can be generated by pressurized gyration. For example, bubbles composed of a spherical core-shell structure are a distinctive platform for numerous healthcare applications including drug delivery, imaging and microbial scavenging. Pressurized gyration offers a new route to form well characterized protein and nanoparticle-decorated protein bubbles [9]. The addition of nanoparticles to the shell of the microbubbles is viable and these impart extra stability and could be optically tuned in infrared and visible wavelength ranges. Also, gold nanoparticle-loaded microbubbles could be used to fabricate quantum dots (Q-dots) that enables bubble imaging in a bimodal manner in both fluorescence and ultrasound. These can also be a promising delivery vehicle for tracking particles in drug delivery and can provide contrast for photoacoustic imaging. Microbubbles prepared with gold nanoparticles showed greater optical extinction values than those without gold nanoparticles and these values increase with the concentration of the gold nanoparticles. They show antibacterial activity against, for example, gram-negative $E$. coli with the bubbles containing the gold nanoparticles performing better than the former [10]. The conjugation of the microbubbles with alkaline phosphatase also allowed detection of, for example, pesticide paraoxon in aqueous solution, and this demonstrates their biosensing capabilities [10]. The active ingredients or drugs could be coated nanoparticles or embedded within the bubble or nanoparticle system, a wide variety of drugs regardless of molecular weight could be loaded and sustainably release enabled at a specific site. The large surface area of the nanoparticles enables high loading content and favors the formation of amorphous dispersion to off-site release [11]. However, more research is needed to control the size and size distribution of bubbles prepared by pressurized gyration if they are to be used in intravenous experiments.

\section{Clinical perspective}

We are living amid an explosion in the life sciences, which has a direct impact on the development of new technologies for the diagnosis, treatment and prevention of human disease. In particular, the birth of new disciplines such as tissue engineering and regenerative medicine, and nanomedicine, is making positive contributions in this sphere.

Several groups of researchers, including clinician-scientists, are working on exciting developments in areas embracing all aspects of patient care from nanotechnology-based theranostic devices for wound healing to tissueengineered solutions for vascular disease. While there is widespread interest in harnessing new technologies in all fields of medicine to realize their full potential, it is perhaps important to prioritize areas in which to focus our research endeavors, nanotechnology being one of them. Clinician involvement from the very outset in this process 
is vital, especially in addressing the persisting areas of 'unmet clinical needs.'

The clinical community is both excited by the promise of what new and emerging technologies can deliver in terms of patient care, and wary of some of the potential dangers of embracing these new fields. Thus, it is important for clinicians, scientists and industry to develop a scientifically and socially conscious platform from which to foster the development of new diagnostics and therapies, within clearly defined and ethically responsible boundaries. Thus, jointly, we should strive to achieve the goal of better healthcare delivery, for all, through nanotechnology.

Financial \& competing interests disclosure

The authors have no relevant affiliations or financial involvement with any organization or entity with a financial interest in or financial conflict with the subject matter or materials discussed in the manuscript. This includes employment, consultancies, honoraria, stock ownership or options, expert testimony, grants or patents received or pending, or royalties.

No writing assistance was utilized in the production of this manuscript.

\section{References}

1 Sosnik A. Production of drug-loaded polymeric nanoparticles by electrospraying technology. J. Biomed. Nanotechnol. 10, 22002217 (2014).

2 Dhar S, Gu FX, Langer R, Farokhzad OC, Lippard SJ. Targeted delivery of cisplatin to prostate cancer cells by aptamer functionalized Pt (IV) prodrug-PLGA-PEG nanoparticles. Proc. Natl Acad. Sci. USA 105, 17356-17361 (2008).

3 Parhizkar M, Reardon PJ, Knowles JC et al. Electrohydrodynamic encapsulation of cisplatin in poly (lactic-co-glycolic acid) nanoparticles for controlled drug delivery. Nanomed. Nanotechnol. Biol. Med. 12, 1919-1929 (2016).

4 Reardon PJ, Parhizkar M, Harker AH et al. Electrohydrodynamic fabrication of core-shell PLGA nanoparticles with controlled release of cisplatin for enhanced cancer treatment. Int. J. Nanomedicine 12, 3913-3926 (2017).

5 Labbaf S, Ghanbar H, Stride E, Edirisinghe M. Preparation of multilayered polymeric structures using a novel four-needle coaxial electrohydrodynamic device. Macromol. Rapid Commun. 35, 618-623 (2014).

6 Parhizkar M, Reardon PJ, Knowles JC et al. Performance of novel high throughput multi electrospray systems for forming of polymeric micro/nanoparticles. Materials \& Design 126, 73-84 (2017).

7 Luo C, Stoyanov SD, Stride E, Pelan E, Edirisinghe M. Electrospinning versus fibre production methods: from specifics to technological convergence. Chem. Soc. Rev. 41, 4708-4735 (2012).

8 Mahalingam S, Edirisinghe M. Forming of polymer nanofibers by a pressurised gyration process. Macromol. Rapid Commun. 34, 1134-1139 (2013).

9 Mahalingam S, Raimi-Abraham BT, Craig DQM, Edirisinghe M. Formation of protein and protein-gold nanoparticle stabilized microbubbles by pressurized gyration. Langmuir 31, 659-666 (2014).

10 Mahalingam S, Xu Z, Edirisinghe M. Antibacterial activity and biosensing of PVA-lysozyme microbubbles formed by pressurized gyration. Langmuir 31, 9771-9780 (2015).

11 Raimi-Abraham BT, Mahalingam S, Davies PJ, Edirisinghe M, Craig DQM. Development and characterization of amorphous nanofiber drug dispersions prepared using pressurized gyration. Mol. Pharm. 12, 3851-3861 (2015). 